\title{
The Difference in Gender Affects the Pathogenesis of Ligamentum Flavum Hypertrophy
}

\author{
Hiroyuki Takashima ${ }^{12)}$, Tsuneo Takebayashi ${ }^{3)}$, Mitsunori Yoshimoto ${ }^{2)}$, Maki Onodera ${ }^{4)}$, Izaya Ogon ${ }^{2)}$, Tomonori Morita ${ }^{2)}$, \\ Noriyuki Iesato $^{2)}$, Yoshinori Terashima ${ }^{2)}$, Katsumasa Tanimoto ${ }^{2)}$ and Toshihiko Yamashita ${ }^{2)}$ \\ 1) Division of Radiology and Nuclear Medicine, Sapporo Medical University Hospital, Sapporo, Japan \\ 2) Department of Orthopedic Surgery, Sapporo Medical University School of Medicine, Sapporo, Japan \\ 3) Sapporo Maruyama Orthopaedic Surgery Hospital, Sapporo, Japan \\ 4) Department of Diagnostic Radiology, Sapporo Medical University School of Medicine, Sapporo, Japan
}

\begin{abstract}
:
Introduction: Gender differences may play a role in the pathogenesis of lumbar spinal stenosis. However, few reports that discuss the effects of gender differences in ligamentum flavum (LF) hypertrophy have been published, and no study has investigated the relationship between LF thickness and the quantitative value of intervertebral disc (IVD) degeneration. This study aimed to investigate the impact of gender on the pathomechanisms underlying LF hypertrophy, focusing on the relationship among LF thickness, IVD degeneration, and age.

Methods: The subjects include 100 patients with low back pain and leg numbness, tingling, or pain. We measured LF thickness and the T2 values of IVDs using MR imaging and analyzed the relationship among LF thickness, T2 values of IVDs, and age. The interclass correlation coefficient (ICC) was calculated as the inter-rater reliability between the LF thick-
\end{abstract} ness values measured by two investigators.

Results: ICC was calculated for the two measurements of LF thickness ( $r=0.923,95 \%$ CI: 0.907-0.936). No statistically significant difference in the T2 values of IVDs was observed between females and males from L2/3 to L5/S. There were significantly negative linear correlations between LF thickness and the T2 values of IVDs at all levels, but this correlation was not observed in females at L4/5. There were significantly negative linear correlations between age and the T2 values of IVDs from L2/3 to L5/S for all patients, females, and males $(r=0.422-0.756)$. In addition, there were significantly positive linear correlations between age and LF thickness from L2/3 to L4/5 for all patients $(r=0.329-0.361)$ and females $(r=$ 0.411-0.481). Correlations were not observed for males at all levels or for all patients at L5/S.

Conclusions: The relationships identified among LF thickness, age, and IVD degeneration suggest that gender differences play a role in the pathogenesis of LF hypertrophy.

Keywords:

Ligamentum flavum hypertrophy, gender, intervertebral disc degeneration, T2 value

Spine Surg Relat Res 2018; 2(4): 263-269 dx.doi.org/10.22603/ssrr.2017-0069

\section{Introduction}

The spine comprises intervertebral discs (IVDs) that provide anterior support, facet joints that provide posterior support, and ligaments that surround the spine. Repeated mechanical stress causes hypertrophy of the facet joints; degenerative changes in the cartilage, IVDs, and osseous structures; and thickening of the ligamentum flavum (LF) and other ligaments ${ }^{1)}$. Lumbar spinal stenosis (LSS) is a common cause of low back and lower extremity pain, particularly in elderly patients ${ }^{2,3}$, and LF hypertrophy is a major contributor to the development of LSS. LF hypertrophy is attributed to increased mechanical stress caused by IVD degeneration with aging ${ }^{4,5}$. However, the detailed mechanism underlying LF hypertrophy remains unclear. Previous studies have suggested the effect of estradiol, a female sex hormone, as a factor potentially related to the pathomechanisms underlying LF hypertrophy ${ }^{6,7)}$. Ishimoto et $a{ }^{8}{ }^{8)}$ reported that the preva-

Corresponding author: Hiroyuki Takashima, takashima@sapmed.ac.jp

Received: September 27, 2017, Accepted: January 22, 2018, Advance Publication: April 7, 2018

Copyright (C) 2018 The Japanese Society for Spine Surgery and Related Research 
Table 1. Distribution of the Examinees with Regard to the Age and Body Mass Index (BMI).

\begin{tabular}{ccccc}
\hline Characteristics & Total & Female & Male & P value \\
\hline Number of subjects & 100 & 48 & 52 & $0.689^{\mathrm{a}}$ \\
Age & $60.3 \pm 16.7$ & $60.4 \pm 16.9$ & $60.1 \pm 16.7$ & $0.978^{\mathrm{b}}$ \\
Mean $\pm \mathrm{SD}$ & & & & \\
BMI $\left(\mathrm{kg} / \mathrm{m}^{2}\right)$ & $23.9 \pm 3.4$ & $23.7 \pm 3.6$ & $24.1 \pm 3.1$ & $0.932^{\mathrm{b}}$ \\
Mean $\pm \mathrm{SD}$ & & & & \\
\hline
\end{tabular}

${ }^{a}$ Chi-square test

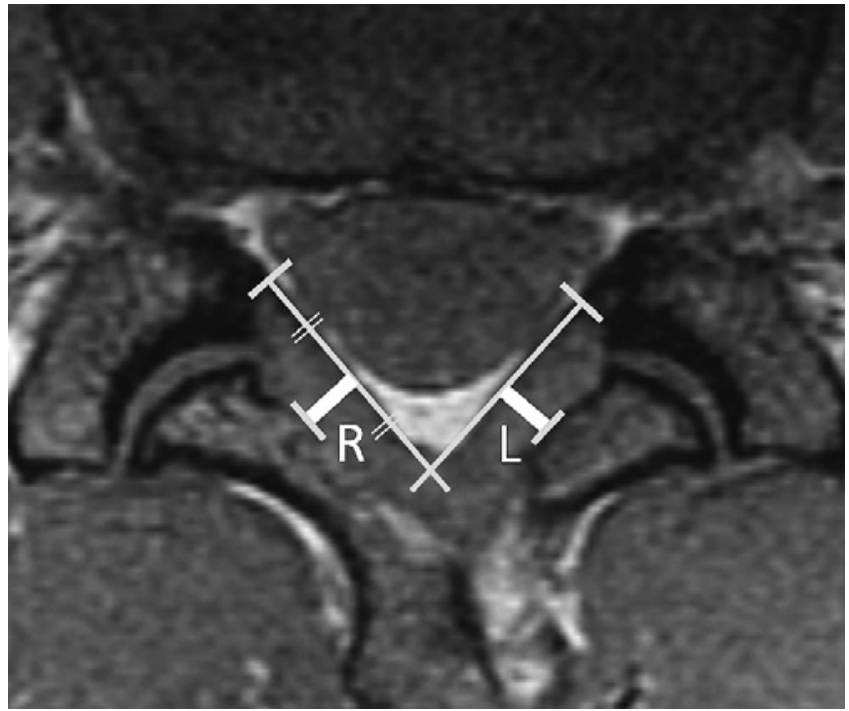

Figure 1. Measurement of ligamentum flavum thickness on axial T1-weighed magnetic resonance images at the facet joint level.

lence of symptomatic LSS in Japanese females significantly increases with age, whereas that in males differs little with age $>60$ years. Furthermore, Yabuki et al. ${ }^{9)}$ reported that the prevalence of LSS in males and females aged 70-79 years was $10.3 \%$ and $11.2 \%$, respectively. These results suggest that gender differences play a role in the pathogenesis of LSS. However, no report that discusses the effects of gender differences on LF hypertrophy has been published.

This study aimed to investigate the effect of gender on the pathomechanisms underlying LF hypertrophy focusing on the relationship among LF thickness, IVD degeneration, and age.

\section{Materials and Methods}

The institutional review board of our institution approved this prospective cross-sectional study, and written informed consent was obtained from the study participants. The study comprised 100 patients aged 23-83 years (44 females and 56 males) who underwent magnetic resonance (MR) examination of the lumbar spine because of low back pain and leg numbness, tingling, or pain (Table 1). The exclusion criteria were as follows: (i) prior spine surgery; (ii) systemic inflam-

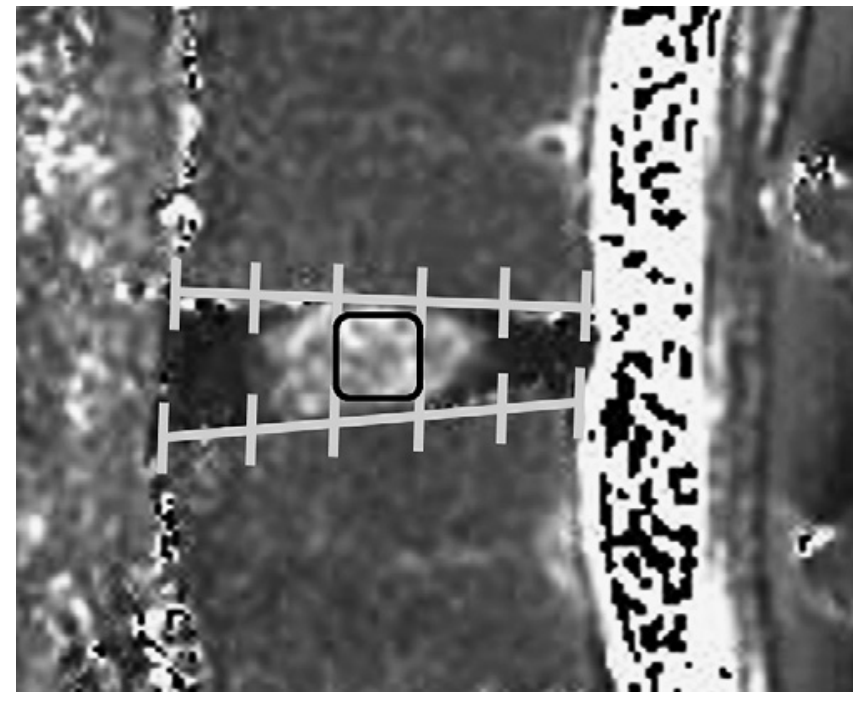

Figure 2. Measurement of $\mathrm{T} 2$ values of the intervertebral discs.

The intervertebral discs (IVDs) were divided into five equal areas, with the middle fifth of the disc designated as the $\mathrm{T} 2$ value of IVD.

matory disease; (iii) neurologic disorder; (iv) acute trauma, neoplasm, or infection; (v) spinal deformities or spondylolisthesis (over Meyerding grade II); and (vi) diabetes. The rationale for the exclusion of patients with diabetes was the association between LF thickness and diabetes reported in previous studies ${ }^{10,11}$. The equipment comprised a spine coil with a GE Signa HDx 1.5 T (GE Healthcare, Milwaukee, WI, USA). We measured LF thickness and T2 values of IVDs at the intervertebral level from L2/3 to L5/S, at which more than $95 \%$ of decompression surgery was performed as previously reported $^{1)}$. The LF thickness was measured on axial T1-weighed MR images (TR/TE: 500 ms/10 ms, FOV: $14 \mathrm{~cm}$, matrix: $256 \times 192$, slice thickness: $4 \mathrm{~mm}$ ) at the facet joint level based on the study reported by Safak et al. ${ }^{1)}$ (Fig. 1). A diagnostic radiologist (M.O.) with 15 years of experience and a research scientist (H.T.) with 12 years of experience in analyzing spine MR images blindly and independently measured all LFs using an electronic ruler with $0.1 \mathrm{~mm}$ resolution. Thickness at the middle portion of LF was measured, and the mean values of both sides were recorded at each level. The interclass correlation coefficient 


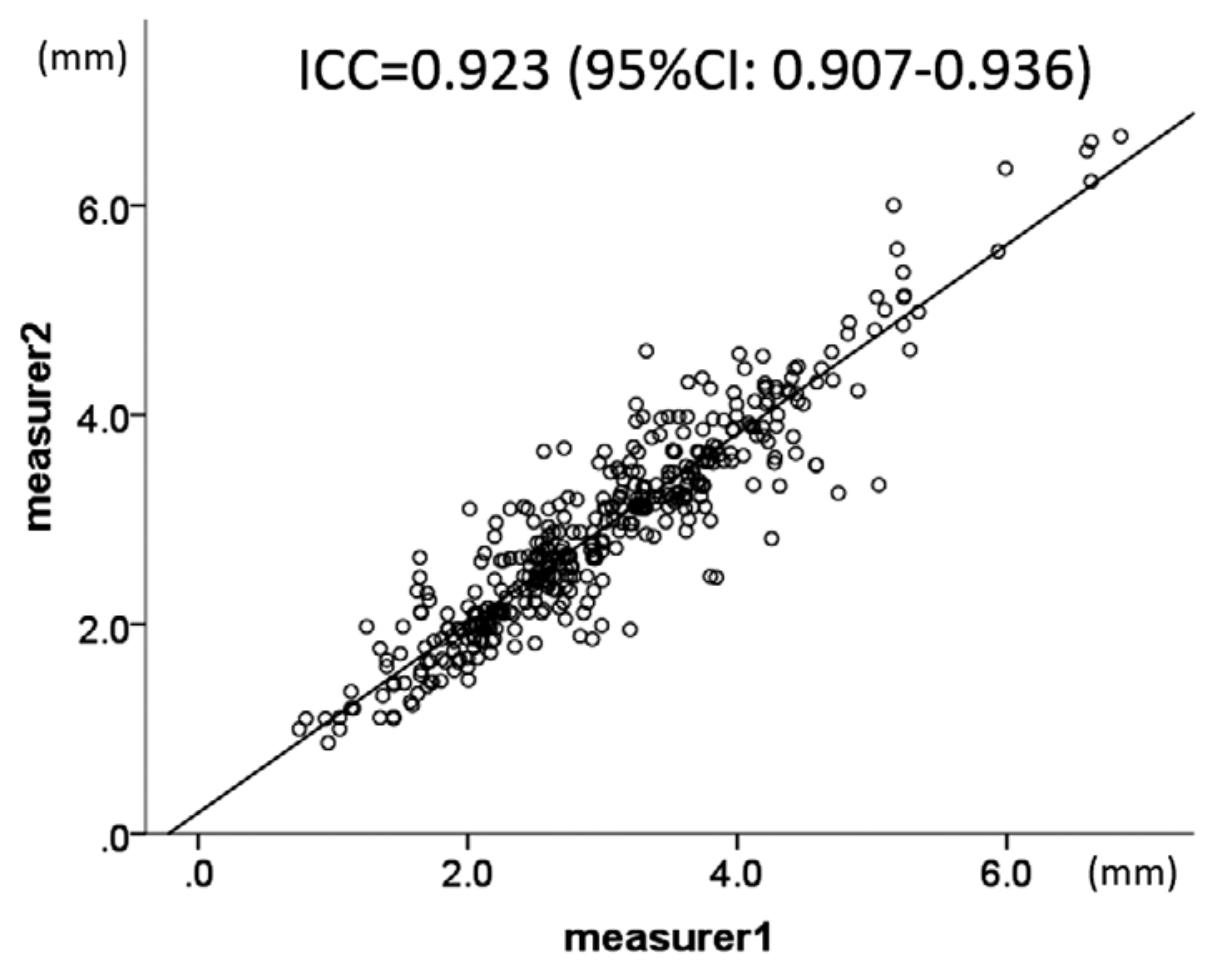

Figure 3. The correlation of LF thickness between investigators 1 and 2, ICC was 0.923.

Table 2. Thickness of Ligamentum Flavum (LF) and T2 Value of Intervertebral Disc (IVD).

\begin{tabular}{lccccc}
\hline & level & All patients & Female & Male & P value $^{\mathrm{a}}$ \\
\hline Flavum thickness mean \pm SD $(\mathrm{mm})$ & L2/3 & $2.56 \pm 0.74$ & $2.43 \pm 0.66$ & $2.59 \pm 0.89$ & 0.313 \\
& L3/4 & $2.81 \pm 0.85$ & $2.72 \pm 0.88$ & $2.89 \pm 0.82$ & 0.305 \\
& L4/5 & $3.31 \pm 1.01$ & $3.27 \pm 0.93$ & $3.34 \pm 1.08$ & 0.710 \\
& L5/S & $3.51 \pm 1.15$ & $3.27 \pm 0.93$ & $3.34 \pm 1.08$ & 0.644 \\
\hline T2 value mean \pm SD (ms) & L2/3 & $75.5 \pm 24.9$ & $71.3 \pm 25.7$ & $79.3 \pm 23.7$ & 0.107 \\
& L3/4 & $73.6 \pm 24.5$ & $71.1 \pm 25.2$ & $75.8 \pm 23.8$ & 0.335 \\
& L4/5 & $71.7 \pm 23.5$ & $69.2 \pm 21.7$ & $74.0 \pm 25.0$ & 0.302 \\
& L5/S & $73.1 \pm 18.4$ & $71.7 \pm 18.7$ & $74.4 \pm 18.3$ & 0.235 \\
\hline
\end{tabular}

${ }^{\mathrm{a}}$ Mann-Whitney U test

Table 3. The Correlation Coefficient Value between Thickness of Ligamentum Flavum and T2 Value of Intervertebral Disc, and Subject Age and T2 Value of IVD, Thickness of LF.

\begin{tabular}{ccccc}
\hline & & All patients & female & male \\
\hline L2/3 & Flavum thickness/T2 value of NP & $-0.694(\mathrm{p}<0.001)$ & $-0.649(\mathrm{p}<0.001)$ & $-0.781(\mathrm{p}<0.001)$ \\
& Age/T2 value of NP & $-0.597(\mathrm{p}<0.001)$ & $-0.756(\mathrm{p}<0.001)$ & $-0.432(\mathrm{p}=0.001)$ \\
& Age/Flavum thickness & $0.361(\mathrm{p}<0.001)$ & $0.466(\mathrm{p}=0.001)$ & $0.286(\mathrm{p}=0.070)$ \\
\hline L3/4 & Flavum thickness/T2 value of NP & $-0.354(\mathrm{p}<0.001)$ & $-0.435(\mathrm{p}=0.002)$ & $-0.298(\mathrm{p}=0.032)$ \\
& Age/T2 value of NP & $-0.614(\mathrm{p}<0.001)$ & $-0.655(\mathrm{p}<0.001)$ & $-0.576(\mathrm{p}<0.001)$ \\
& Age/Flavum thickness & $0.329(\mathrm{p}=0.001)$ & $0.481(\mathrm{p}=0.01)$ & $0.160(\mathrm{p}=0.256)$ \\
\hline \multirow{2}{*}{ L4/5 } & Flavum thickness/T2 value of NP & $-0.373(\mathrm{p}<0.001)$ & $-0.275(\mathrm{p}=0.058)$ & $-0.451(\mathrm{p}=0.001)$ \\
& Age/T2 value of NP & $-0.441(\mathrm{p}<0.001)$ & $-0.456(\mathrm{p}=0.001)$ & $-0.440(\mathrm{p}=0.001)$ \\
& Age/Flavum thickness & $0.331(\mathrm{p}=0.001)$ & $0.411(\mathrm{p}=0.004)$ & $0.265(\mathrm{p}=0.072)$ \\
\hline \multirow{2}{*}{ L5/S } & Flavum thickness/T2 value of NP & $-0.448(\mathrm{p}<0.001)$ & $-0.488(\mathrm{p}<0.001)$ & $-0.430(\mathrm{p}=0.001)$ \\
& Age/T2 value of NP & $-0.454(\mathrm{p}<0.001)$ & $-0.486(\mathrm{p}<0.001)$ & $-0.422(\mathrm{p}=0.002)$ \\
& Age/Flavum thickness & $0.228(\mathrm{p}=0.053)$ & $0.282(\mathrm{p}=0.052)$ & $0.197(\mathrm{p}=0.161)$ \\
\hline
\end{tabular}


All patients

(a)

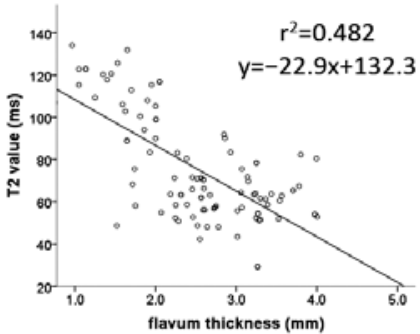

(b)

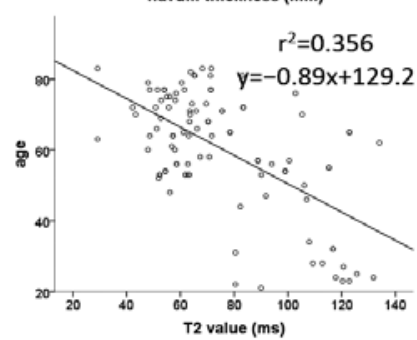

(c)

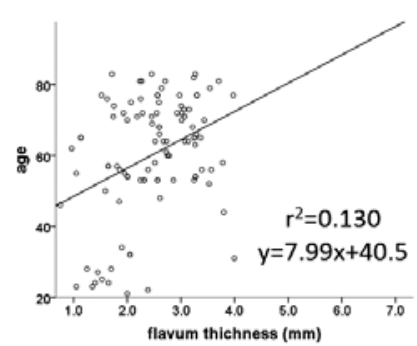

female

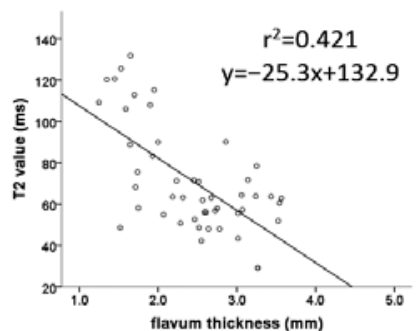

$r^{2}=0.571$
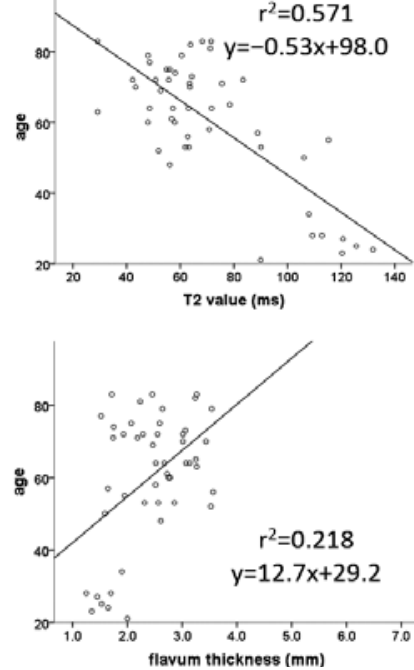

male

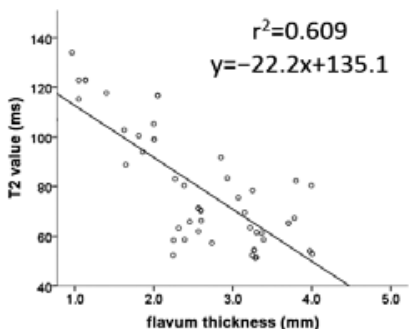

$r^{2}=0.186$
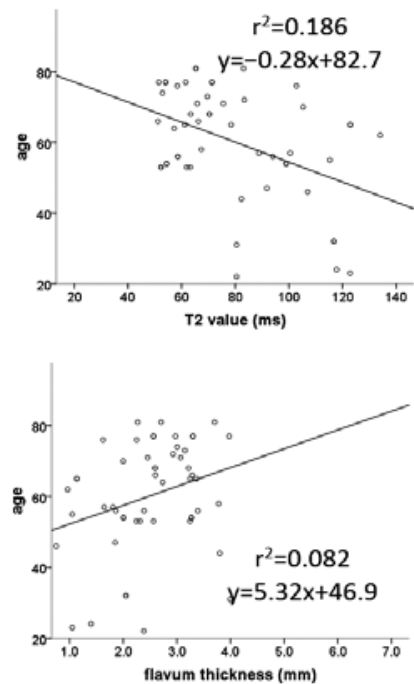

Figure 4. Correlation between (a) ligamentum flavum (LF) thickness and the T2 values of intervertebral discs (IVDs), (b) T2 value of IVDs and age, (c) LF thickness and age at the level of L2/3 in all patients, only females, and only males.

All patients

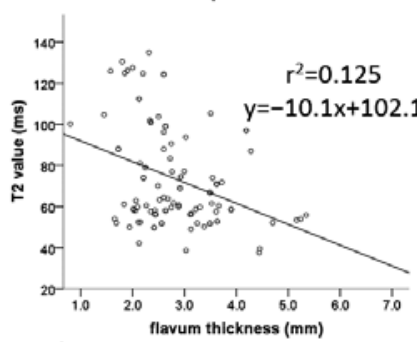

(b)

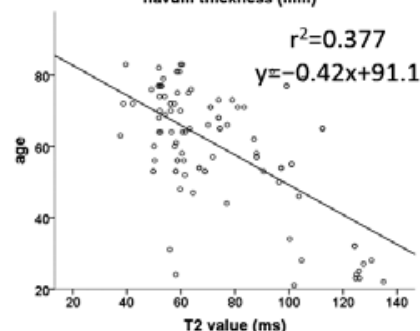

(c)

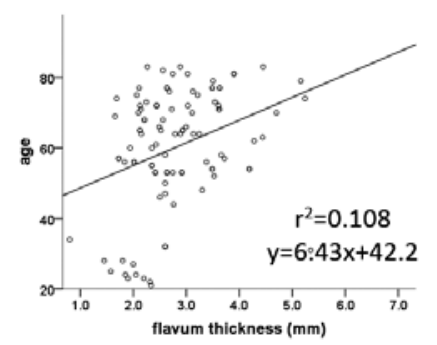

female
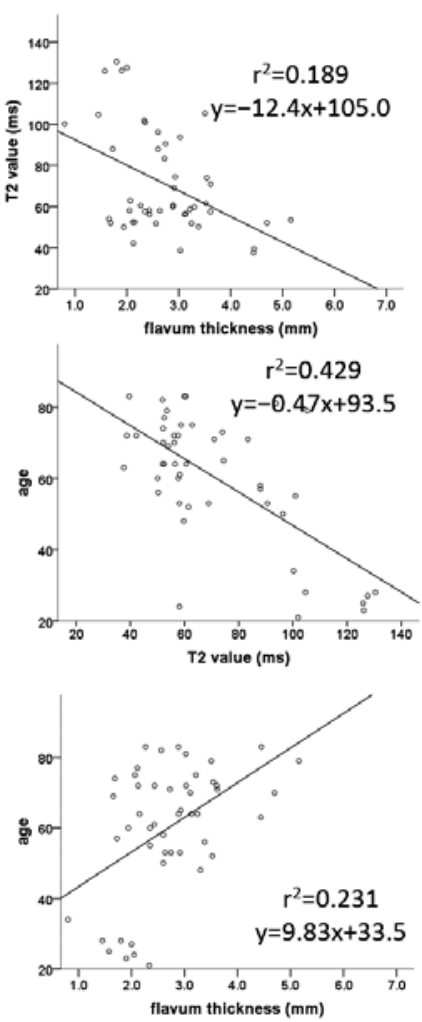

male
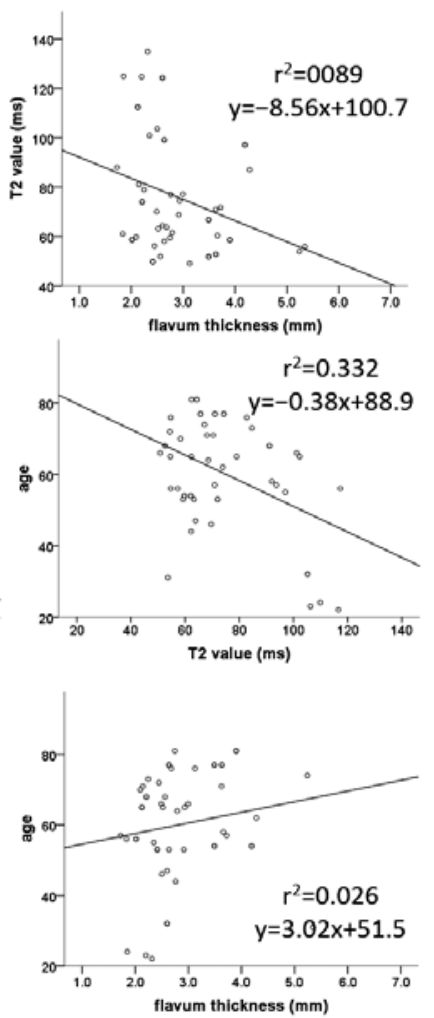

Figure 5. Correlation between (a) ligamentum flavum (LF) thickness and the $\mathrm{T} 2$ value of intervertebral discs (IVDs), (b) T2 values of IVDs and age, (c) LF thickness and age at the level of L3/4 in all patients, only females, and only males. 
(a)

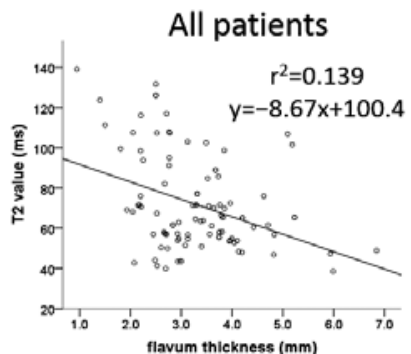

(b)

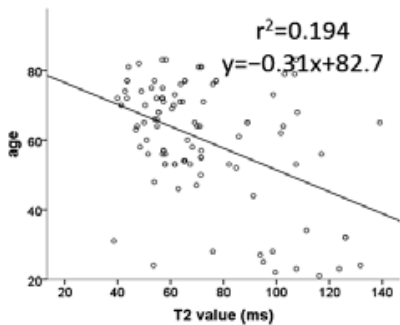

(c)

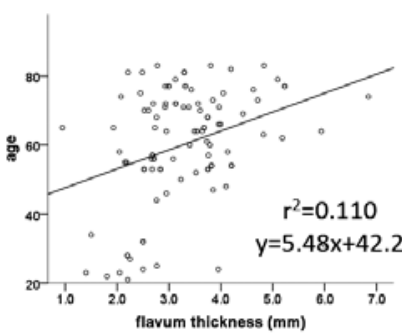

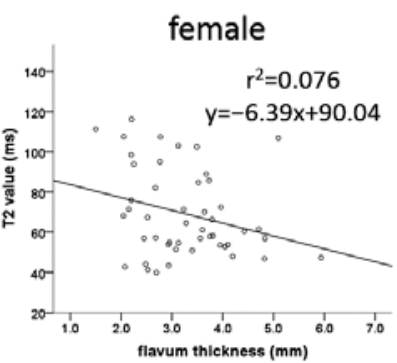
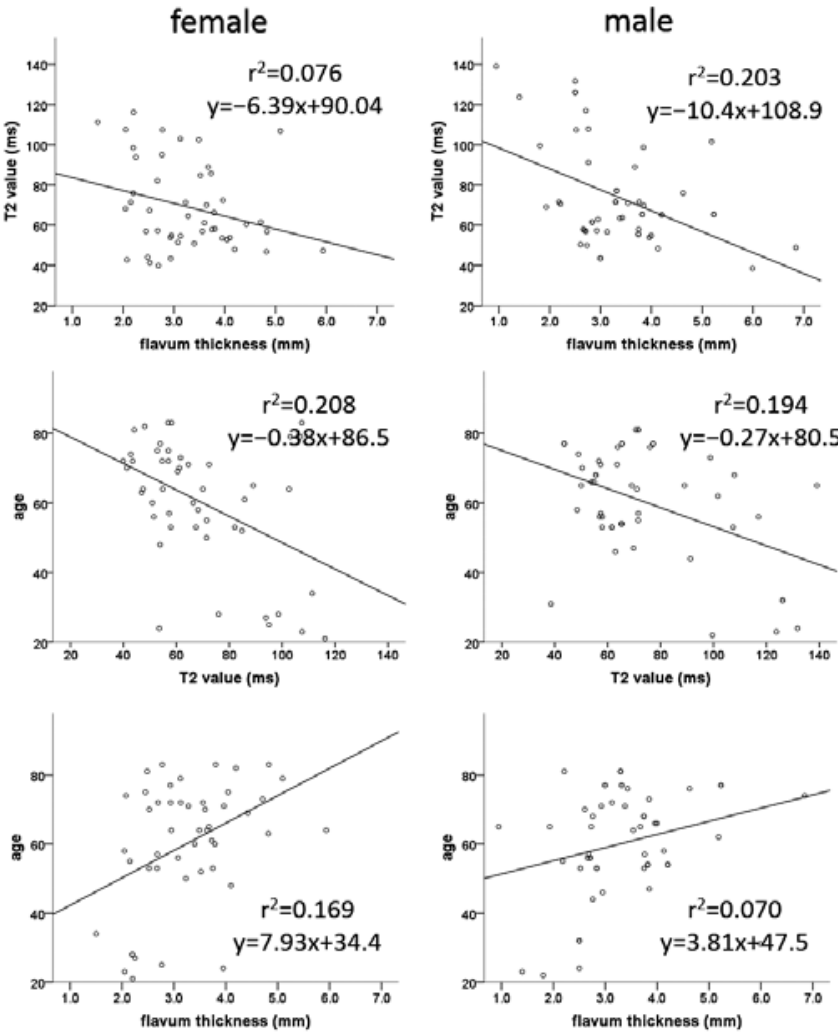

Figure 6. Correlation between (a) ligamentum flavum (LF) thickness and the T2 values of intervertebral discs (IVDs), (b) T2 values of IVDs and age, (c) LF thickness and age at the level of L4/5 in all patients, only females, and only males.

All patients

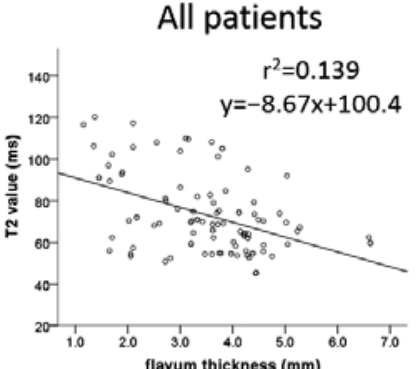

(b)

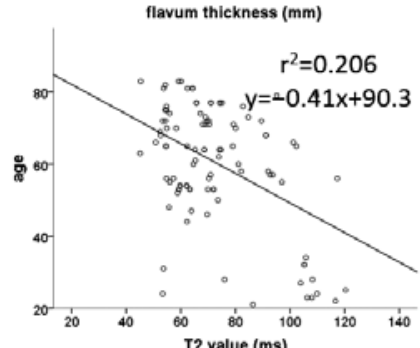

(c)

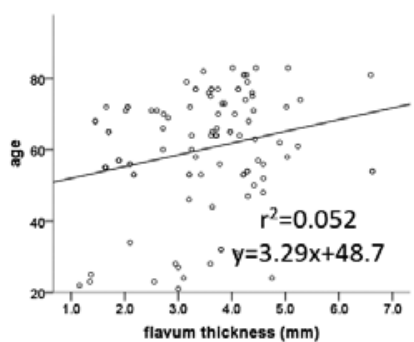

female
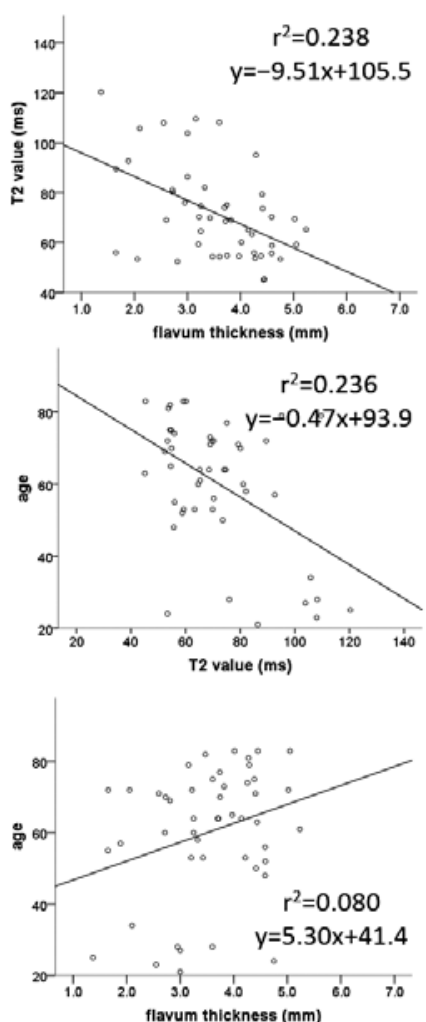

male
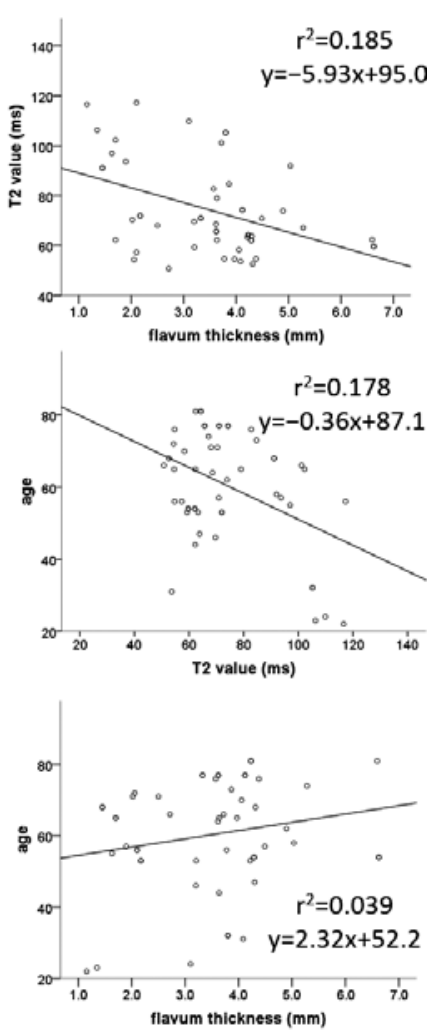

Figure 7. Correlation between (a) ligamentum flavum (LF) thickness and the T2 values of intervertebral discs (IVDs), (b) T2 values of IVDs and age, (c) LF thickness and age at the level of L5/S1 in all patients, only females, and only males. 
(ICC) was calculated as the inter-rater reliability between the LF thickness values measured by the two investigators.

A T2 map was created using T2 values in the midsagittal section from the sagittal sections centered on the lumbar midline region with optimized eight multi-spin echoes (TR/ first echo TE, last echo TE: 1,000/14.8 ms, $118.6 \mathrm{~ms}$; RBW: $\pm 15.63 \mathrm{kHz}$; FOV: $22 \mathrm{~cm}$; matrix: $320 \times 256$; slice thickness/gap: 4/4 mm; 5 slices; NEX 2; total scan time: 8 min and $34 \mathrm{~s}$ ) obtained using an Advantage Workstation (version 4.4, Functool; GE Healthcare, Milwaukee, WA, USA). However, the first echo from the multi-spin system was excluded to minimize the effect of the stimulated echo. A T2 map was generated for each pixel from the signal intensity (SI) in the respective TE using the following calculating formula: $\mathrm{SI}=\mathrm{e}^{-\mathrm{TE} / \mathrm{T} 2}$

For measurements, IVDs were divided into five equal areas, with the middle fifth of IVD designated as the T2 value of $\mathrm{IVD}^{12}$. The mean values in the regions of interest were semi-automatically measured by a research scientist (H.T.) with 12 years of experience in analyzing spine MR images (Fig. 2). We analyzed the relationships among LF thickness, T2 value of IVD, and age using Pearson's correlation coefficient test. A p value of $<0.05$ was considered statistically significant. Statistical analyses were performed using SPSS for Windows, version 20.0 (SPSS Inc., Chicago, IL, USA).

\section{Results}

ICC was calculated between the two LF thickness values ( $r=0.923,95 \%$ CI: $0.907-0.936)$ measured by the two researchers (Fig. 3). LF thickness and the T2 values of IVD at each level are shown in Table 2. No statistically significant differences in the T2 values of IVDs were observed between females and males. LF thickness was also observed between females and males. The $r$ values for LF thickness and the T2 values of IVDs, age and the T2 values of IVDs, and age and LF thickness are shown in Table 3. The $r$ values for LF thickness and the T2 value of IVD at L2/3 were -0.694 ( $p<$ $0.001)$ for all patients, $-0.649(\mathrm{p}<0.001)$ for females, and -0.781 ( $p<0.001$ ) for males (Fig. 4). Similarly, L3/4 were $-0.354(\mathrm{p}<0.001),-0.435(\mathrm{p}=0.002)$, and -0.298 ( $\mathrm{p}=$ 0.032), respectively (Fig. 5). However, the corresponding values for $\mathrm{L} 4 / 5$ were -0.373 ( $\mathrm{p}<0.001),-0.275$ ( $\mathrm{p}=$ $0.058)$, and $-0.451(\mathrm{p}=0.001)$, indicating no significant correlation in females (Fig. 6). The $\mathrm{r}$ values for $\mathrm{L} 5 / \mathrm{S}$ were -0.448 ( $\mathrm{p}<0.001),-0.488(\mathrm{p}<0.001)$, and $-0.430(\mathrm{p}=$ 0.001 ), respectively (Fig. 7). There were significantly negative correlations between age and the T2 values of IVDs from $\mathrm{L} 2 / 3$ to $\mathrm{L} 5 / \mathrm{S}$ for all patients, females, and males $(\mathrm{r}=$ $0.422-0.756)$. There were significantly positive correlations between subject age and LF thickness from L2/3 to L4/5 for all patients $(\mathrm{r}=0.329-0.361)$ and females $(\mathrm{r}=0.411-0.481)$ but not for males at all levels or all patients at L5/S.

\section{Discussion}

LF hypertrophy was first reported in 1913 as a major cause of spinal stenosis leading to low back and lower extremity pain ${ }^{1,3}$. LF hypertrophy is attributed to increased mechanical stress caused by IVD degeneration with aging. In fact, previous studies ${ }^{2,5,13)}$ have reported a relationship between LF thickness and IVD degeneration with aging. Altinkaya et al. ${ }^{5)}$ and Munns et al. ${ }^{13)}$ reported a significant increase in LF thickness with increasing IVD degeneration. However, Sakamaki et al. ${ }^{2}$ observed no correlation between LF thickness and IVD degeneration. This inconsistency may be due to the lack of repeatability and objectivity in the visual evaluation of IVD degeneration based on Pfirrmann classification $^{12,14-16)}$. Therefore, we quantitatively analyzed IVD degeneration using MRI T2 mapping, which allows the quantification of decreasing water content in IVD with increasing IVD degeneration ${ }^{12,17}$. To the best of our knowledge, few studies have investigated the relationship between LF thickness and the quantitative value of IVD degeneration.

The results of the current study indicated a stronger correlation between the LF thickness and the T2 values of IVDs than between LF thickness and age. Therefore, we concluded that mechanical stress is the most important factor that influences LF hypertrophy. In particular, from $\mathrm{L} 2 / 3$ to $\mathrm{L}$ $5 / \mathrm{S} 1$ in males, and L5/S1 in both females and males, the effect of mechanical stress seems to be dominant because there were no statistical correlations between age and LF thickness. No correlation was found between LF thickness and IVD degeneration at L4/5 in females, but a correlation was found between LF thickness and age. There are only a few reports on the effect of gender on LF thickness. Abbas et al. ${ }^{4)}$ and Safak et al. ${ }^{1)}$ reported no difference in LF thickness between genders. The findings of the present study were consistent with those of these previous studies. However, when we focused on the correlation among LF thickness, age, and IVD degeneration, a difference in the mode of LF hypertrophy was observed between genders. Previous studies reported that normal LF comprises $80 \%$ elastic fibers and $20 \%$ collagen fibers and that hypertrophic LF exhibits a loss of elastic fibers and increase in collagen fibers, resulting in increased collagen-to-elastin ratio ${ }^{18)}$. In an in vitro study, Chen et al. ${ }^{19)}$ reported that estradiol regulated MMP13 expression via the PI3 L pathway and that the collagento-elastin ratio in the cell culture media decreased after LF cells were treated with estradiol. Therefore, LF hypertrophy in females may be affected by decreased estradiol level because of the aging process and menopause. There have been no reports on the effects of other sex hormones. In contrast, increased mechanical stress on LF caused by IVD degeneration appears to be a more dominant factor than age for LF hypertrophy in males. Therefore, the onset of instability including spondylolisthesis and lumbar canal stenosis may be related to gender differences ${ }^{20,21)}$.

The present study has several limitations that should be 
addressed. First, the number of subjects was relatively less and the population had a deviation in age. Second, we were unable to investigate the time of menopause and estradiol level in each patient. Thus, we cannot deny the possibility that these factors affected the results. Third, all participants had low back or lower extremity pain, indicating that the results might be different in the general population. Finally, we measured the thickness of the LF center alone. Some studies have reported that the mechanical stress is greater on the posteromedial side of LF than on the anterolateral $\operatorname{side}^{7,13)}$. In the future, measuring LF thickness by region will enable more detailed evaluation of the pathomechanisms underlying LF hypertrophy.

In conclusion, the results of the present study demonstrated the relationships among LF thickness, age, and IVD degeneration. These results suggested that the differences play a role in the pathogenesis of LF hypertrophy and the onset of instability including spondylolisthesis and lumbar canal stenosis may be related to gender differences.

Conflicts of Interest: The authors declare that there are no relevant conflicts of interest.

Author Contributions: Hiroyuki Takashima wrote and prepared the manuscript, and all of the authors participated in the study design. All authors have read, reviewed, and approved the article.

\section{References}

1. Safak AA, Is M, Sevinc O, et al. The thickness of the ligamentum flavum in relation to age and gender. Clin Anat. 2010;23(1):79-83.

2. Sakamaki T, Sairyo K, Sakai T, et al. Measurements of ligamentum flavum thickening at lumbar spine using MRI. Arch Orthop Trauma Surg. 2009;129(10):1415-9.

3. Zhong ZM, Zha DS, Xiao WD, et al. Hypertrophy of ligamentum flavum in lumbar spine stenosis associated with the increased expression of connective tissue growth factor. J Orthop Res. 2011;29 (10): $1592-7$

4. Abbas J, Hamoud K, Masharawi YM, et al. Ligamentum flavum thickness in normal and stenotic lumbar spines. Spine. 2010;35 (12): 1225-30

5. Altinkaya N, Yildirim $\mathrm{T}$, Demir $\mathrm{S}$, et al. Factors associated with the thickness of the ligamentum flavum: is ligamentum flavum thickening due to hypertrophy or buckling? Spine. 2011;36(16):E 1093-7.

6. Okuda T, Baba I, Fujimoto Y, et al. The pathology of ligamentum flavum in degenerative lumbar disease. Spine. 2004;29(15):168997.

7. Sairyo K, Biyani A, Goel V, et al. Pathomechanism of ligamentum flavum hypertrophy: a multidisciplinary investigation based on clinical, biomechanical, histologic, and biologic assessments. Spine. 2005;30(23):2649-56.

8. Ishimoto Y, Yoshimura N, Muraki S, et al. Prevalence of symptomatic lumbar spinal stenosis and its association with physical performance in a population-based cohort in Japan: the Wakayama Spine Study. Osteoarthr Cartil. 2012;20(10):1103-8.

9. Yabuki S, Fukumori N, Takegami M, et al. Prevalence of lumbar spinal stenosis, using the diagnostic support tool, and correlated factors in Japan: a population-based study. J Orthop Sci. 2013;18 (6):893-900.

10. Lotan R, Oron A, Anekstein Y, et al. Lumbar stenosis and systemic diseases: is there any relevance? J Spinal Disord Tech. 2008; 21(4):247-51.

11. Asadian L, Haddadi K, Aarabi M, et al. Diabetes Mellitus, a New Risk Factor for Lumbar Spinal Stenosis: A Case-Control Study. Clin Med Insights Endocrinol Diabetes. 2016;9:1-5.

12. Takashima H, Takebayashi T, Yoshimoto M, et al. Correlation between T2 relaxation time and intervertebral disk degeneration. Skeletal Radiol. 2012;41(2):163-7.

13. Munns JJ, Lee JY, Espinoza Orias AA, et al. Ligamentum flavum hypertrophy in asymptomatic and chronic low back pain subjects. PLoS One. 2015;10(5):e0128321.

14. Watanabe A, Benneker LM, Boesch C, et al. Classification of intervertebral disk degeneration with axial $\mathrm{T} 2$ mapping. AJR Am J Roentgenol. 2007;189(4):936-42.

15. Takebayashi T, Takashima H, Yoshimoto M. Analysis of Degenerative Discs in Lumbar Spondylolisthesis Using MRI T2 Mapping. Open J Radiol. 2012;2(03):77.

16. Takashima H, Takebayashi T, Yoshimoto $M$, et al. Investigation of Intervertebral Disc and Facet Joint in Lumbar Spondylolisthesis using T2 Mapping. Magnetic resonance in medical sciences : MRMS : an official journal of Japan Society of Magnetic Resonance in Medicine. 2014;13(4):261-6.

17. Ogon I, Takebayashi T, Takashima H, et al. Analysis of chronic low back pain with magnetic resonance imaging T2 mapping of lumbar intervertebral disc. J Orthop Sci. 2015;20(2):295-301.

18. Kosaka H, Sairyo K, Biyani A, et al. Pathomechanism of loss of elasticity and hypertrophy of lumbar ligamentum flavum in elderly patients with lumbar spinal canal stenosis. Spine. 2007;32(25): 2805-11.

19. Chen MH, Hu CK, Chen PR, et al. Dose-dependent regulation of cell proliferation and collagen degradation by estradiol on ligamentum flavum. BMC musculoskeletal disorders. 2014;15:238.

20. Jacobsen S, Sonne-Holm S, Rovsing H, et al. Degenerative lumbar spondylolisthesis: an epidemiological perspective: the Copenhagen Osteoarthritis Study. Spine. 2007;32(1):120-5.

21. Uribe JS, Beckman J, Mummaneni PV, et al. Does MIS Surgery Allow for Shorter Constructs in the Surgical Treatment of Adult Spinal Deformity? Neurosurgery. 2017;80(3):489-97.

Spine Surgery and Related Research is an Open Access journal distributed under the Creative Commons Attribution-NonCommercial-NoDerivatives 4.0 International License. To view the details of this license, please visit (https://creativeco mmons.org/licenses/by-nc-nd/4.0/) 